\title{
ÊPHÊMẼROPTẼRES DU SUD-OUEST DE LA FRANCE.
}

\section{I. - MigRations dimagos a haUte ALtitude}

\author{
par A. G. B. Thomas ${ }^{1}$.
}

\begin{abstract}
Une revue bibliographique des travaux concernant les migrations d'imagos d'insectes aquatiques vers l'amont des cours d'eau est donnée. De telles migrations sont étudiées sur les Ephéméroptères de deux torrents des Pyrénées entre 2160 et $2750 \mathrm{~m}$ d'altitude. Plus de 800 imagos $q$ de Rhithrogena loyolaea Navás et de Baetis alpinus (Pictet) ont été récoltées dans l'eau, après oviposition dans des zones nettement en amont de la limite de présence des larves âgées. Le déplacement des $q$ vers l'amont se fait jusqu'au point de jaillissement de l'eau : source froide ou limite de l'enneigement du torrent, ce qui confirme l'exactitude de l'hypothèse du cycle de colonisation de Müller chez les Ephéméroptères de haute montagne. Des gains d'altitude d'un minimum de $170 \mathrm{~m}$ (B. alpinus) et de $350 \mathrm{~m}$ ( $R$. loyolaea) ont été observés. L'éclosion peut avoir lieu, chez $B$. alpinus, dans des caux dont la température est toujours comprise entre 0 et $3^{\circ} \mathrm{C}$.
\end{abstract}

\section{Ephemeroptera of Southwest France :}

\section{Migrations of adults at high altitude.}

Work on the upstream migrations of adults of aquatic insects is reviewed. The migrations of Ephemeroptera were studied in two Pyrenean streams at altitudes between 2160 and $2750 \mathrm{~m}$. About 800 adult females of Rhithrogena loyolaea Navás and Baetis alpinus (Pictet) have been collected in the water, after oviposition in the zone situated clearly upstream from the area containing the old larvae. The upstream movement of females occurs up to the point at which the water gushes out : a cold source or the limit of snow cover for the stream. These results, therefore, exactly confirm the colonisation cycle hypothesis of Müller for the Ephemeroptera of high mountains. For a few imagines, the minimum gain in altitude was $170 \mathrm{~m}$ ( $B$. alpinus, and $350 \mathrm{~m}$ ( $R$. loyolaea). Hatching of $B$. alpinus can take place in water with temperatures always between 0 and $3^{\circ} \mathrm{C}$.

L'hypothèse du cycle de colonisation de Müller (1954) supposs un mécanisme antagoniste de la dérive des larves d'insectes aquatiques vers l'aval : la migration des imagos vers l'amont. Oviposition puis éclosion dans les zones les plus élevées des ruisseaux compenseraient ainsi le dépeuplement progressif dû à la dérive.

L'imporlance qualitative et quantilative de cette migration vers l'amont, et surtout ses rapports avec la dérive, ont été depuis mis en doute - voire contestés — entre autres par Einsele:2 (1960),

1. Laboratoire d'Hydrobiologie, Université Paul-Sabatier, 118, route de Narbonne, 31077 Toulouse Cedex, France.

2. Cité par Russev (1970). 
Waters (1965), Elliott (1967), Schwoerbel (1969), Hynes (1970 b), Schumacher (1970) et Bishop (1973). Indubitablement, jusqu'à ces dernières années, l'attention a surtout été retenue par les mouvements des larves.

Chez les Ephéméroptères, en haute montagne du moins, l'importance des vols d'imagos o vers les sources des torrents paraît cependant avoir été sous-estimée jusqu'ici. Or, la remontée par voie aérienne semble, dans bien des cas, être la plus efficace par suite des trois faits suivants :

- des obstacles gênent la progression ascendante des larves (grandes cascades ; substrat localement défavorable, à peu près dépourvu d'abris, comme la roche en place) ;

- des crues violentes, soudaines el fréquentes, provoquent des dérives catastrophiques sur substrat instable (cailloutis des cirques glaciaires par exemple). Certains œufs, en grands nombres et souvent solidement fixés aux pierres, peuvent mieux y résister que les larves ;

-. la température de l'eau, issue de sources froides ou de névés et toujours inféricure à 3 ou $4{ }^{\circ} \mathrm{C}$, ralentit l'activité des larves et limite la densité des populations.

\section{I. - REVUE BIBLIOGRAPHIQUE}

Si l'on fait le point sur les connaissances actuelles à ce sujet, la situation paraît assez confuse.

La tendance des imagos $\&$ d'Éphéméroplères à voler vers l'amont a été signalée à plusieurs reprises et depuis longtemps, notamment par Stadler in Ulmer (1927), Schoenemund (1930), Sawyer (1950), Harris (1952) et Bogoescu et Tabacaru (1969). Schoenemund, en particulier, pense déjà à un mécanisme compensateur de la dérive : "Flug der Erhaltung des Nachwuchses ... ». Mais toutes ces observations demeurent sommaires et le processus éventuel n'est pas démontré.

De l'élude de densités de populations larvaires, Dorris et Copeland (1962) déduisent l'existence des migrations remontantes d'imagos. Mais leur raisonnement n'exclut pas la possibilité de mouvements de larves vers l'amont - mouvemen!s susceptibles de variations importantes selon les caractères physiques des stations et les stades larvaires - et ne tient pas compte non plus d'éventuels écarts dans les taux de mortalité, ce que Macan avait exprimé auparavant (1957).

Cette remontée des imagos n'est pas particulière aux Ephéméroptères ; clle a également été observée chez d'autres ordres d'insectes aquatiques: Plécoptères (Muttkowski 1929ㄹ, Schwarz 1970), Tri-

1. Cité par Hynes (1970a). 
choptères (Waters 1968, Elliott 1969 et 1971, Pearson et Kramer 1972). Elle a été présumée chez les Diptères Simuliidae (Davies et coll. 1962, Mackay 1969).

Mais en définitive, peu de travaux ont été consacrés exclusivement aux mouvements des imagos d'insectes aquatiques.

Roos (1957) a été le premier à tenter d'illustrer le cycle de colonisation. A l'aide de pièges dérivés de celui de Malaise (1937), il a montré un vol vers l'amont assez net chez plusieurs espèces lotiques de Trichoptères et, dans une certaine mesure, de Plécoptères el d'Éphéméroptères du genre Baetis.

Russev (1959, 1973) a mis en évidence sur un fleuve un mouvement vers l'amont d'imagos d'Êphéméroptères appartenant à plusieurs espèces, en particulier Palingenia longicauda. Son interprétation est celle d'une compensation de la dérive des œufs entre le moment où ils touchent la surface de l'eau et celui où ils sont immobilisés sur le fond (profondeur moyenne du cours d'eau étudié : $10 \mathrm{~m}$ ).

Par piégeage, Müller (1973) a constaté une migration, en général remontante (plus de $90 \%$ des imagos) du Trichoptère Philopotamus montanus. Il utilisait en particulier des pièges à écran (Chapman et Kinghorn 1955). Par marquage et recapture, Nishimura (1967) a montré la remontée, vers l'amont d'une rivière japonaise, du Trichoptère Stenopsyche griseipennis.

Bengtsson el coll. (1972) puis Madsen et coll. (1973) ont utilisé des écrans adhésifs sur les deux faces, tendus transversalement au-dessus du cours d'eau. Ils ont ainsi mis en évidence une nette tendance à la remontée de plusieurs espèces d'Ephéméroptères et d'une espèce de Plécoptères. Ces auteurs, ainsi que Schwarz (1970), ont d'autre part observé sur des imagos marquées, un déplacement vers l'amont et une tendance à se rapprocher de la source, respectivement, de Brachyptera risi et Isoperla goertzi (Plécoptères).

Mais on peut dire qu'une partie de la définition du cycle de colonisation de Müller : «..oviposition always occurs at the upper limit of the natural habitat», seulement très probable chez les Plécoptères, reste finalement encore à établir, surtout pour les autres groupes. En toute rigueur, migration vers l'amont n'implique pas oviposition. Svensson (1974) a pu constater ainsi, le long d'un cours d'eau, des remontées d'imagos de Noctuidae qui n'ont certainement aucun rapport avec le cycle de colonisation ! Et des migrations massives de l'Odonate Libellula quadrimaculata vers l'amont de l'Escaut et de ses affluents seraient en rapport avec la présence d'un Trématode parasite (Dumont et Hinnekint, 1973).

De plus, le cheminement des imagos peut être lent (déplacements terrestres), complexe et très éloigné d'un trajet direct vers 
l'amont ainsi que l'ont observé plusieurs auteurs à la suite dı travail de Thomas (1966) sur le Plécoptère Capnia atra. Lehmann (1970) a, pour sa part, relevé chez Philopotamus montanus des nombres d'imagos supéricurs en aval, avec toutefois, en amont, une majorité de $q$ ayant déjà pondu.

Svensson (1974), dans une étude sur le vol des Trichoptères, a montré qu'en moyenne, sur 15 espèces, $55 \%$ des 9 se déplaçaient vers l'amont et $45 \%$ vers l'aval, celles de deux espèces seulement atteignant de façon significative la proportion de $60 \%$ vers l'amont.

Les résultats de certains travaux vont même à l'encontre de l'hypothèse du cycle de colonisation de Müller. Ainsi, Elliott (1967) pense qu'un vent vers l'amont est nécessaire. Schumacher (1970) montre que chez le genre Hydropsyche (Trichoptères), les déplacements d'imagos se font autant vers l'aval que vers l'amont, tandis que le mécanisme de compensation paraît exclusivement aquatique. De son côte, Bishop (1973) indique des vols d'Ephéméroptères, Plécoptères et Trichoptères nettement orientés vers l'aval !

Ces contradictions suggèrent quelques remarques :

- en ce qui concerne les Ephéméroptères, tout déplacement d'imago devra être interprété avec prudence, à cause de la fréquence des comportements erratiques comme celui, observé à plusieurs reprises par divers auteurs, à l'origine de pontes aberrantes sur des automobiles mouillées, des routes humides (Ferago 1937', Lestage 1937, Spieth 1940, Harris 1952 et observations personnelles) ou sèches, façades de bâtiments, etc. (Verrier 1941, 1954, 1956).

- les modalités et l'amplitude des remontées des imagos vers l'amont des cours d'eau varient beaucoup d'un groupe taxonomique à l'autre. Mais il n'est pas certain qu'on puisse séparer nettement les insectes aquatiques en deux catégories (ceux qui accomplissent le cycle de colonisation et ceux qui ne l'accomplissent pas) sans intermédiaires comme le fait Müller (1973). Chez beaucoup d'espèces, de Trichoptères en particulier, la dispersion des q lors du vol de ponte paraît être élevée (Svensson 1974). Les nombres ou les pourcentages d'individus migrant vers l'amont ou vers l'aval, cités par les différents auteurs, ne tiennent pas comple ou guère compte - des migrations latérales, extrêmement difficiles à évaluer, qui risquent d'introduire, dans bien des cas, des marges d'erreurs très importantes.

Il n'est même pas exclı que le comportement d'espèces voisines puisse être assez différent. A priori, les migrations d'imagos vers l'amont pourraient concerner en particulier les espèces fortement exposées à la dérive et ne possédant guère, à l'état larvaire, de

1. Cité par Lestage (1937). 
mécanisme cfficace de compensation, situation aggravée lorsque la production est faible.

Les caractères physiques du cours d'eau et de son environnement ont ecrtainement aussi une influence sur l'existence du mécanisme ou tout au moins sur son efficacité.

- à ma connaissance, aucune étude axée sur de lelles migrations chez les insectes aquatiques n'a été effectuée jusqu'ici en haute monlagne (nettement plus de $2000 \mathrm{~m}$ d'altitude).

\section{II. - MẼTHODE EMPLOYẼE}

Ce travail a été effectué à haute altitude sur deux torrents d'accès long el difficile, et même pratiquement impossible l'hiver pour le second. Les influences humaines directes sont done nulles. Ces deux cours d'eau ont été également retenus pour leur isolement (cirques glaciaires, pas d'autres ruisseaux dans les environs) : sauf migration à très grande distance, les imagos récoltées ne peutvent provenir que des torrents étudiés.

36368 invertébrés aquatiques ont été récoltés dont 11622 Ephéméroptères (845 imagos et 10777 larves). L'étude des migrations a élé limitée aux Êphéméroptères dont la vie imaginale très brève exclut tout déplacement pour une recherehe de nourriture.

Les imagos of n'ont jamais été capturées en vol nuptial mais pendant el surtoul après la ponte. Il n'a done été utilisé aucune technique de piégeage.

La technique de marquage el recapture est, à priori, très séduisante pour les études de migrations. Elle paraît difficilement applicable aux adultes d'Éphéméroptères, d'abord en raison de la fragilité de ceux-ci, ensuite à cause de la nécessité de marquer un grand nombre de specimens, ce qui est peu compatible avec les conditions de récolte de ces insectes à haute altitude (durée de vie très brève). En effel, les pourcentages recapturés sont toujours faibles ; en voici deux exemples concernant des insecles beaucoup plus robustes que les Éphéméroptères adultes et de récolte plus aisée (durée de vie imaginale de plusieurs semaines) :

- 1,6\% de recaptures sur 1500 specimens marqués du Plécoptère Brachyptera risi pour des migralions comprises entre 50 et $250 \mathrm{~m}$ de distance seulement (Madsen et coll. 1973);

- nettement moins de $1 \%$ sur plus de 75000 Diptères Brachycères marqués, à près de $2000 \mathrm{~m}$ d'altitude, pour une migration de $3,6 \mathrm{~km}$ de distance, malgré des moyens de recapture considérables (Aubert, Goeldlin et Lyon 1969).

Dans le présent travail, en un premier temps, j'ai pu observer à plusieurs reprises en été, sur un cours d'eau issu d'une source 
très froide, la remontée de nombreuses imagos $q$ jusqu'au point de jaillissement de l'eau.

La seconde partie a été effectuée sur un torrent formé par la confluence de ruisseaux issus de névés. Elle constitue un essai de mesure de la distance parcourue par les imagos. La méthode utilisée est fort simple : des imagos $q$ ayant pondu sont récoltées dans les prélèvements de faune benthique, bien en amont de la limite de présence des larves âgées. La distance mesurée correspond ainsi à une migration minimale effectuée par les imagos.

Les deux espèces d'Ephéméroptères étudiées sont Baetis alpinus (Pictet 1843) et Rhithrogena loyolaea Navás 1922. Elles ont été décrites en détail, respectivement, par Müller-Liebenau (1969) et Thomas (1970).

\section{III. - MISE EN EVIDENCE DU CYCLE DE COLONISATION : RUISSEAU DE LAVEDAN}

Le ruisseau de Lavedan, long de $11,5 \mathrm{~km}$ est un affluent de la rive gauche de la Neste d'Aure. Il est issu à $2230 \mathrm{~m}$ d'une source froide, enneigée en moyenne de novembre à juillet.

- Cette source constitue la première station étudiée. Sur quelques mètres, la pente et la profondeur sont faibles, le fond est constitué de pierres moyennes ou grosses avec des touffes de Graminées, de Saxifrages et de Bryophytes. Des imagos d'Ephéméroptères ont été récoltées à la fois dans les prélèvements benthiques quantitatifs effectués pendant la période de déneigement (tableau 1) et par chasse à vue entre les pierres du lit, jamais à plus de $\mathbf{2} \mathbf{m}$ de distance du point de jaillissement de l'eau (tableau 2).

Une analyse de l'eau, prélevée en période d'étiage (le 24-X-1973), a donné les résultats suivants, exprimés en $\mathrm{mg} / \mathrm{l}$ :

$\begin{array}{cccccccc}\mathrm{H} \mathrm{CO}_{3}{ }^{-} & \mathrm{Ca}^{++} & \mathrm{NO}_{3}-\mathrm{N} & \mathrm{SiO}_{2} & \mathrm{So}_{4}{ }^{--} & \mathrm{K}^{+} & \mathrm{Mg}^{++} & \mathrm{Na}^{+} \\ 22 & 7,8 & 0,27 & 2,17 & 1 & 0,2 & 0,12 & 0,22\end{array}$

Résistivité : $\chi_{20}=41 \mu \mathrm{mhos} / \mathrm{cm}^{2} / \mathrm{cm}$.

Cette eau est donc très peu minéralisée; le contenu ionique dépend essenticllement des teneurs en bicarbonate et en calcium.

La température de l'eau ne dépasse pas $3{ }^{\circ} \mathrm{C}$ en plein été.

- Seconde station à $150 \mathrm{~m}$ en aval de la précédente (alt. $2180 \mathrm{~m}$ ) après une pente moyenne de $30 \%$. Nombreuses touffes de Bryophytes, et sur les bords, de Graminées et de Saxifrages. La température de l'eau a atteint $6^{\circ} \mathrm{C}$ en août 1972 .

- Troisième station à $150 \mathrm{~m}$ encore en aval (alt. $2160 \mathrm{~m}$ ). La pente est réduite ( 5 à $10 \%$ ), le cours bien plus large $(4-5 \mathrm{~m})$, la 


\begin{tabular}{|c|c|c|c|c|c|c|c|c|c|c|c|c|c|c|}
\hline & $\begin{array}{l}5 \\
5 \\
0\end{array}$ & & $\frac{n}{n}+\frac{a}{2}$ & $\begin{array}{l}\bar{\sigma} \\
\dot{y}\end{array}$ & $+\frac{0}{n}$ & $\begin{array}{l}\infty \\
\infty \\
\end{array}$ & ल & & n & & & $\begin{array}{l}\infty \\
0\end{array}$ & $\begin{array}{l}m \\
0 \\
\infty\end{array}$ & E d \\
\hline $\begin{array}{l}\text { E } \\
0 \\
m \\
\text { N }\end{array}$ & $\frac{\pi}{* \frac{\pi}{x}}$ & $\begin{array}{l}\frac{5}{5} \\
\frac{10}{0} \\
\text { in } \\
\text { in }\end{array}$ & $\bar{\sigma}$ & $\begin{array}{c}- \\
\infty\end{array}$ & mo & $\begin{array}{l}\infty \\
\sigma \\
\sigma\end{array}$ & $\sigma$ & m & $\stackrel{n}{-}$ & 5 & & $\stackrel{\varphi}{-}$ & $\begin{array}{l}m \\
n \\
N\end{array}$ & 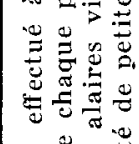 \\
\hline $\begin{array}{l}\text { N } \\
\ddot{0} \\
0 \\
0 \\
2\end{array}$ & $\begin{array}{r}\frac{N}{\alpha} \\
* \frac{x}{N} \\
\frac{x}{N}\end{array}$ & $\begin{array}{l}i \\
i \\
\frac{0}{2}\end{array}$ & 0 & $\bar{a}$ & $-\underline{0}$ & $\begin{array}{l}0 \\
\infty \\
\end{array}$ & L & o & & & & & $\overline{-}$ & 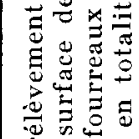 \\
\hline $\begin{array}{l} \pm \\
\\
u\end{array}$ & $\begin{array}{l}\text { N } \\
\text { 三 } \\
\equiv \\
\equiv \\
D^{\prime}\end{array}$ & $\begin{array}{l}1 \\
0 \\
0 \\
0\end{array}$ & $\bar{q}$ & $\begin{array}{c}\infty \\
n \\
n\end{array}$ & $m$ & $\begin{array}{l}n \\
m\end{array}$ & $r$ & a & & ก & & $\begin{array}{l}0 \\
10\end{array}$ & & 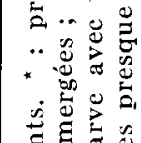 \\
\hline $\begin{array}{l}\alpha \\
\supset \\
0 \\
\infty\end{array}$ & 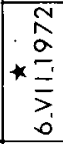 & $\begin{array}{l}\frac{1}{0} \\
\frac{1}{0} \\
\stackrel{N}{N}\end{array}$ & $\bar{N}$ & 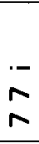 & I & $\infty$ & s & $=$ & & - & & $\nabla$ & $\stackrel{0}{-}$ & 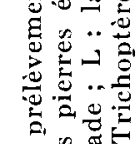 \\
\hline $\begin{array}{l}\dot{1} \\
\vdots \\
2 \\
2 \\
0 \\
\dot{0}\end{array}$ & 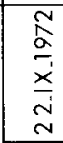 & $\begin{array}{l}\frac{f}{8} \\
\dot{0} \\
\dot{0}\end{array}$ & 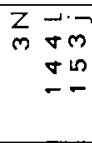 & $\begin{array}{l}Z \\
0 \\
N\end{array}$ & 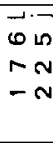 & $\begin{array}{l}\text { A } \\
\text { A }\end{array}$ & $n$ & & & & & & $\begin{array}{l}0 \\
0 \\
\text { N }\end{array}$ & 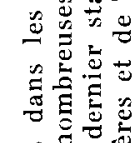 \\
\hline $\begin{array}{l}- \\
\\
0\end{array}$ & $\begin{array}{l}2 \\
N \\
a \\
\bar{\vdots} \\
\bar{\equiv} \\
\infty\end{array}$ & $\begin{array}{l}= \\
= \\
0 \\
0\end{array}$ & $\begin{array}{r}z-1.0 \\
-100\end{array}$ & $Z_{10}$ & $\begin{array}{l}\vec{H} \\
r-9\end{array}$ & $\frac{\infty}{m}$ & $\begin{array}{l}a \\
\text { is } \\
-\end{array}$ & & & & & & & 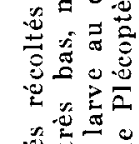 \\
\hline $\begin{array}{l}\varepsilon \\
0 \\
10 \\
-\end{array}$ & 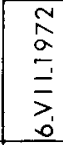 & $\begin{array}{l}-5 \\
0 \\
0 \\
0 \\
0\end{array}$ & $\begin{array}{l}\vec{D} \cdot \\
\infty N \\
-n \\
--\end{array}$ & $\bar{N}$ & $\begin{array}{l}\vec{\infty} \infty \\
0 N \\
-\end{array}$ & $\begin{array}{l}\text { V } \\
0 \\
\end{array}$ & $\stackrel{c}{c}$ & & & & & $\infty$ & $\begin{array}{l}n \\
n \\
n\end{array}$ & 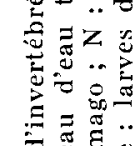 \\
\hline $\begin{array}{l}0 \\
U \\
\text { L } \\
3 \\
0 \\
0\end{array}$ & $\frac{N}{a}$ & $\begin{array}{l}\text { I } \\
\text { in } \\
\text { in }\end{array}$ & 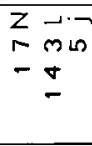 & $\bar{z}$ & $\begin{array}{l}\frac{N}{-N} \\
=-1\end{array}$ & $\stackrel{n}{\infty}$ & 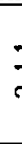 & 7 & & & 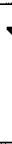 & & $\begin{array}{l}\boldsymbol{\infty} \\
\mathbf{N}\end{array}$ & 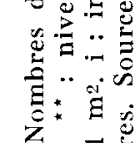 \\
\hline $\begin{array}{l}- \\
0 \\
0\end{array}$ & 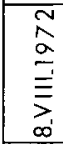 & $\begin{array}{l}= \\
= \\
\text { in } \\
\text { i }\end{array}$ & 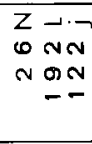 & $\begin{array}{l}Z \\
m \\
- \\
=\end{array}$ & $\begin{array}{l}m \cdot \\
m m \\
m m\end{array}$ & 5 & ? & & & & & $\stackrel{0}{-}$ & & 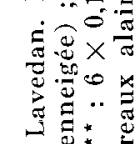 \\
\hline $\begin{array}{l}E \\
0 \\
0 \\
M\end{array}$ & $\begin{array}{l}\text { na } \\
a \\
\vdots \\
= \\
\vdots \\
0\end{array}$ & $\begin{array}{l}\frac{1}{6} \\
\frac{0}{0} \\
\text { in } \\
8\end{array}$ & 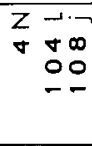 & & $\begin{array}{l}-10 \\
\nabla 0 \\
\text { v in } \\
\text { mo }\end{array}$ & $\hat{m}$ & 0 & & & & & $\nabla$ & $\begin{array}{l}0 \\
\nabla\end{array}$ & 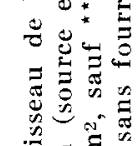 \\
\hline $\begin{array}{l}n \\
z \\
0 \\
\frac{1}{\leftarrow} \\
\leftarrow \\
\text { s }\end{array}$ & $\begin{array}{l}\sim \\
w \\
\leftarrow \\
\square \\
0\end{array}$ & 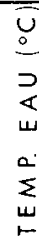 & $\begin{array}{ll} & 0 \\
& 0 \\
& 0 \\
0 & z \\
c & z \\
0 & 0 \\
0 & 0 \\
0 & 0 \\
\frac{2}{5} & 0 \\
:= & 0 \\
\frac{1}{\alpha} & 0\end{array}$ & $\frac{n}{2}$ & $\begin{array}{l}5 \\
\frac{5}{0} \\
\frac{5}{0} \\
0 \\
2 \\
\frac{0}{a} \\
\frac{0}{0}\end{array}$ & $\frac{0}{0}$ & 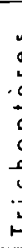 & & $\begin{array}{l}\infty \\
\$ \\
0 \\
0 \\
0 \\
0 \\
0 \\
0 \\
0 \\
0\end{array}$ & 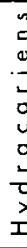 & & $\begin{array}{l}0 \\
0 \\
0 \\
0 \\
\leftarrow \\
0 \\
E \\
\Phi \\
Z\end{array}$ & $\begin{array}{l}n \\
0 \\
\frac{n}{0} \\
\frac{0}{a} \\
\frac{0}{a}\end{array}$ & 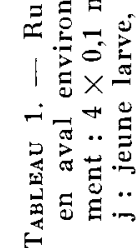 \\
\hline
\end{tabular}


profondeur faible. Le fond, à peu près dépourvu de Bryophytes, Graminées et Saxifrages, est constitué de pierres plates. La température de l'eau a atteint $10,5^{\circ} \mathrm{C}$ lors de l'été 1972 . Le réchauffement de l'eau est donc rapide.

Ces températures ont été relevées sur des thermomètres à maximaminima immergés pendant tout l'été. 22017 invertébrés, dont 5006 larves et 744 imagos d'Ephéméroptères, ont été récoltés. Chaque prélèvement $\left(0,4 \mathrm{~m}^{2}\right)$ résulte de la somme de 4 prélèvements particls de $0,1 \mathrm{~m}^{2}$ réalisés avec un filet de Surber de $0,2 \mathrm{~mm}$ de vide de maille.

La grande fragilité des imagos d'Ephéméroplères, rapidement détruites dans l'eau et en général entraînées à la première pluie, a rendu leur récolte assez aléatoire. Par exemple, la chasse du $3-I X-1974$, effectuéc 24 heures après un orage n'a fourni aucune imago, à l'exclusion de celles trouvées dans le prélèvement benthique.

Cependant, les tableaux 1 et 2 prouvent une remontée d'imagos de $R$. loyolaea et $B$. alpinus jusqu'à la souree. L'amplitude de ce déplacement est au moins égale à $300 \mathrm{~m}$ chez $B$. alpinus et à $150 \mathrm{~m}$ chez R. loyolaea au début du mois de juillet.

\begin{tabular}{|c|c|c|c|c|c|c|c|c|}
\hline STATIONS & $\begin{array}{r}300 \mathrm{~m} \\
50 \mathrm{U}\end{array}$ & $\begin{array}{l}a \vee a I \\
R \subset E\end{array}$ & & $\mathbf{s}$ & 0 & $\mathbf{R}$ & (1) & \\
\hline DATES & $5.1 \times .1971$ & $4.1 \times .1973$ & $5.1 \times .1971$ & 6.V1I.1972 & 8.VIII.1972 & $22.1 \times .1972$ & $4.1 \times .1973$ & $24 . X .1973$ \\
\hline $\begin{array}{l}\text { Rhithrogeno } \\
\text { loyoloea }\end{array}$ & 4 & 3 & 22 & neige & 1 & 4 & 8 & 4 \\
\hline $\begin{array}{l}\text { Boetis } \\
\text { alpinus }\end{array}$ & 0 & 0 & 89 & neige & 10 & 128 & 50 & 47 \\
\hline
\end{tabular}

Tabieav 2. - Ruisseau de Lavedan. Nombres d'imagos récoltées à vue dans le lit du ruisseau, à l'exclusion des prélèvements quantitatifs. 1 : à moins de $2 \mathrm{~m}$ en aval du point de jaillissement de l'eau.

\begin{tabular}{|l|c|c|c|c|c|c|}
\hline $\begin{array}{l}\text { DISTANCE A } \\
\text { LA SOURCE }\end{array}$ & $0 \mathrm{~m}$ & $50 \mathrm{~m}$ & $100 \mathrm{~m}$ & $150 \mathrm{~m}$ & $200 \mathrm{~m}$ & $300 \mathrm{~m}$ \\
\hline $\begin{array}{l}\text { TEMP EAU } \\
\text { A I5h }{ }^{\circ} \mathrm{C}\end{array}$ & $2,5^{\circ}$ & $3.5^{\circ}$ & $4^{\circ}$ & $5^{\circ}$ & $7^{\circ}$ & $9^{\circ}$ \\
\hline $\begin{array}{l}\text { Rhithrogeno } \\
\text { loyoloea }\end{array}$ & 0 & 1 & 2 & 8 & 19 & 43 \\
\hline $\begin{array}{l}\text { Baelis } \\
\text { alpinus }\end{array}$ & 0 & 5 & 64 & 55 & 74 & 191 \\
\hline
\end{tabular}

Tableav 3. - Ruisseau de Lavedan. Nombres de larves au dernier stade récoltées par $\mathrm{m}^{2}$ le $4-\mathrm{IX}-1973$.

Au début de septembre 1973 , pour tenir compte des migrations éventuelles des larves âgées vers l'amont (débit faible et tempé- 
rature de l'eau relativenent élevée), des prélèvements ont été effeclués tous les $50 \mathrm{~m}$ à partir de la source sur des surfaces d'un $\mathrm{m}^{2}$. Date et biotope choisis étaient particulièrement favorables à la récolte de larves au dernier stade (légers biefs près des bords, en courant modéré). Le lablcau 3 montre que des déplacements d'imagos des deux espèces vers l'amont sont encore nécessaires - bien que de plus faible amplitude qu'en début de période de vol - pour que l'oviposition ait lieu à la source.

On remarquera (tableau 1) le petil nombre de larves jeunes (j) de $B$. alpinus à la source el l'absence de larves de $R$. loyolaea. Il est possible que la majeure partic des éclosions n'ait pas licu en été ou que la dérive des larves vers des parties du cours d'eau plus chaudes intervienne dès l'éclosion ou peu après (si cette dernière a toutefois lieu chez $R$. loyolaea dans ces conditions extrômes). Il serait nécessaire d'effectuer des prélèvements sous la neige à intervalles réguliers pour préciser ces deux points.

\section{Conclusion.}

Les tableaux 1 à 3 n'apportent aucune précision sur la proportion éventuelle des imagos d'Ephéméroptères qui effectuent une migration vers l'amont. Ils confirment cependant l'exactitude de l'hypothèse de Müller pour Baetis alpinus, on particulier en ce qui concerne la colonisation de «la limite supérieure de l'hahitat naturel » puisque seules de jeunes larves se rencontrent a la source du Lavedan, la plupart mesurant moins de $2 \mathrm{~mm}$ de longueur, cerques non compris.

\section{IV. - ESSAI DE MESURE DE LA DISTANCE PARCOURUE : RUISSEAU DE CAP DE LONG}

Le ruisseau de Cap de Long (Carte 1) est issu du Gourg de Cap de Long à $2845 \mathrm{~m}$. Il esl temporaire sur plus de $500 \mathrm{~m}$ en septembre.

A $2560 \mathrm{~m}$, il reçoil sur la rive gauche deux affluents issus de névés el recouverts par la neige sur une distance variable selon les étés : en 1972, le premier n'a été déneigé que sur quelques dizaines de mètres en amont de la confluence.

Longueurs et pentes moyennes : r. de Cap de Long : $1700 \mathrm{~m}$ et $17 \% ; 1^{\text {er }}$ affluent (r. du milieu) : $900 \mathrm{~m}$ et $10 \% ; 2^{\text {r }}$ affluent (r. de droite) : $950 \mathrm{~m}$ et $19 \%$. Vitesses du courant et débits sont du même ordre mais le $r$. de Cap de Long coule sur du granit à amphibole, les deux autres sur des pélites (schistes). Des touffes de Bryophytes aquatiques se rencontrent par places mais seulement sur le $r$. de Cap de Long (station 9). 


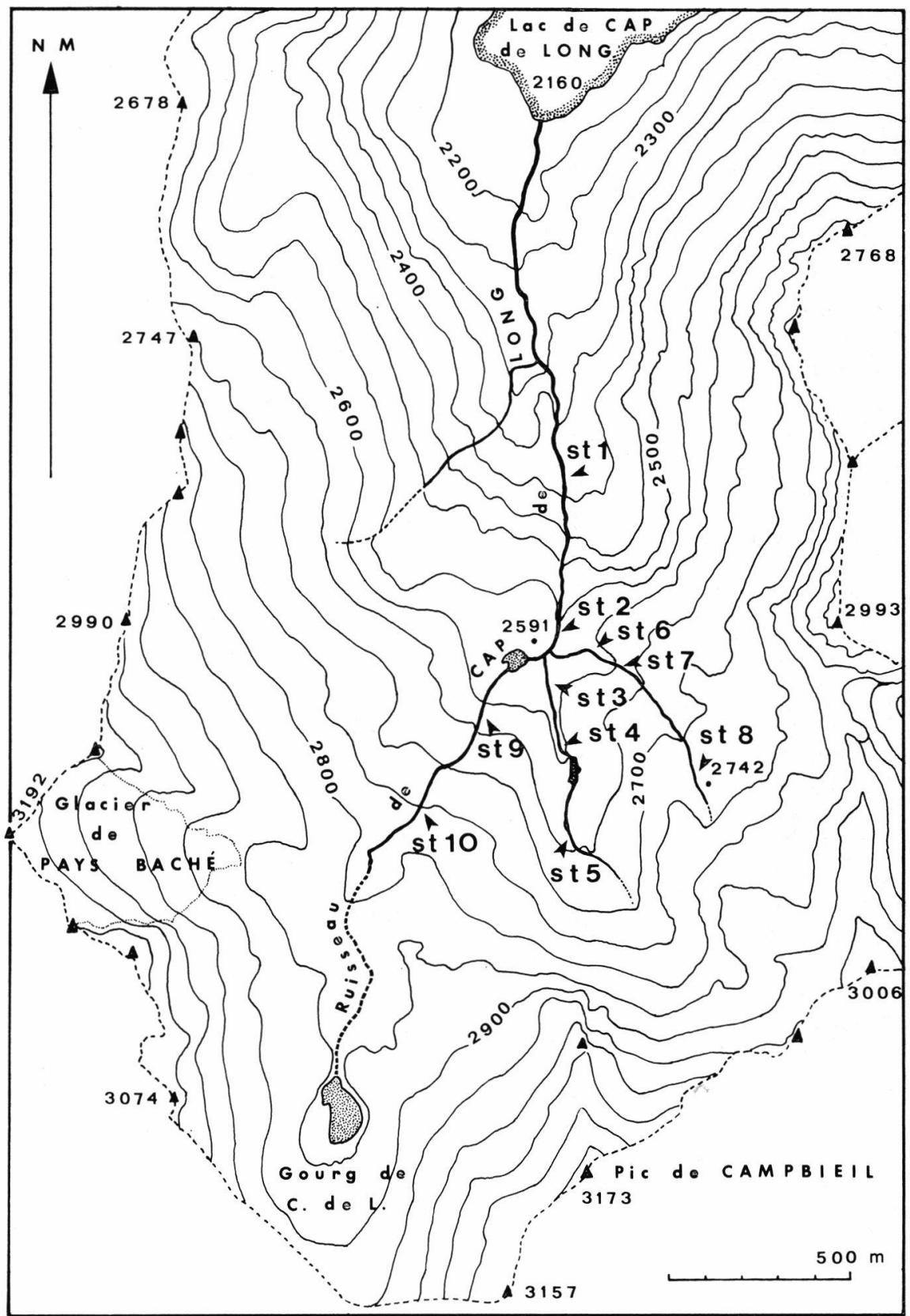

Carte 1. - Le ruisseau de Cap de Long et ses affluents. 
Une trentaine de mètres on aval du confluent, se trouve une cascade d'environ $170 \mathrm{~m}$ de hauteur et $450 \mathrm{~m}$ de longueur (pente moyenne : $38 \%$ ). La station la plus basse (station 1: altitude $2380 \mathrm{~m}$ ) est immédialement en aval. Le cours du torrent se poursuit encore sur plus d'un $\mathrm{km}$ (pente moyenne $18 \%$ ) pour aboutir au lac artificiel de Cap de Isong, retenue de 65,7 millions de $\mathrm{m}^{3}$ à $2160 \mathrm{~m}$.

Une analyse d'eau prélevée en période d'étiage (le 8-IX-1973) à $2560 \mathrm{~m}$, en aval du confluent, a donné les résultats suivants, exprimés en $\mathrm{mg} / \mathrm{l}$ :

$\begin{array}{cccccccc}\mathrm{HCO}_{3}{ }^{-} & \mathrm{Ca}^{++} & \mathrm{NO}_{3}-\mathrm{N} & \mathrm{SiO}_{2} & \mathrm{So}_{4} & \mathrm{~K}^{+} & \mathrm{Mg}^{++} & \mathrm{Na}^{+} \\ 14 & 5,8 & 0,05 & 1 & 2,5 & 0,2 & \mathbf{0 , 1 0} & 0,22\end{array}$

Résistivité : $\chi_{20}=31 \mu \mathrm{mhos} / \mathrm{cm}^{2} / \mathrm{cm}$.

La minéralisation tolale de cette eau est donc très faible.

Les plus fortes températures ont été relevées en IX-1973 par temps chaud et faible débit. Elles peuvent être considérées comme des maxima exceptionnels (Lableau 5) car les deux névés alimentant les ruisseaux du milieu et de droite avaient presque totalement disparu.

10 stations ont été choisies (carte 1 ) et 26 prélèvements non quantitatifs ont été effectués avec un filet troubleau de $0,2 \mathrm{~mm}$ de vide de maille. Les surfaces prospectées à chaque prélèvement ont été très approximativement de $0,1 \mathrm{~m}^{2}$ en 1971 et de $0,5 \mathrm{~m}^{2}$ en 1972 et 1973.

14351 invertébrés dont 5 771 larves et 101 imagos d'Éphéméroptères pondant ou ayant déjà pondu onl été prélevés. Des imagos sont présentes dans les trois ruisseaux nettement en amont (stations 5, 8 et 10) de la limite atteinte par les larves (tableau 4). Les dates de récolte (tableau 5) apportent plus de précision sur les migrations d'imagos, car des mouvemenls de larves âgées vers l'amont ne sont pas exclus, surloul lors des températures maximales (septembre).

Rhithrogena loyolaea ne présente un cycle de développement complet qu'au bas de la cascade (st. $1: 2380 \mathrm{~m}$ ). L'amplitude minimale des migrations (observées sur 18 imagos) est comprise entre 400 et $1200 \mathrm{~m}$ pour un gain d'altitude atteignant $350 \mathrm{~m}$. Mais il n'a été dénombré lors des prélèvements que 6 larves au dernier stade - pour 2 à $3 \mathrm{~m}^{2}$ - à la station 1. La plupart des imagos récoltées viennent donc vraisemblablement de plus bas, entre 2250 et $2160 \mathrm{~m}$ d'altitude où les densités de population sont supérieures et les captures de larves au dernier stade bien plus abondantes. S'il en est bien ainsi, les distances de migration et les gains d'altitude sont respectivement de l'ordre de 1 à $2 \mathrm{~km}$ et de $500 \mathrm{~m}$. 


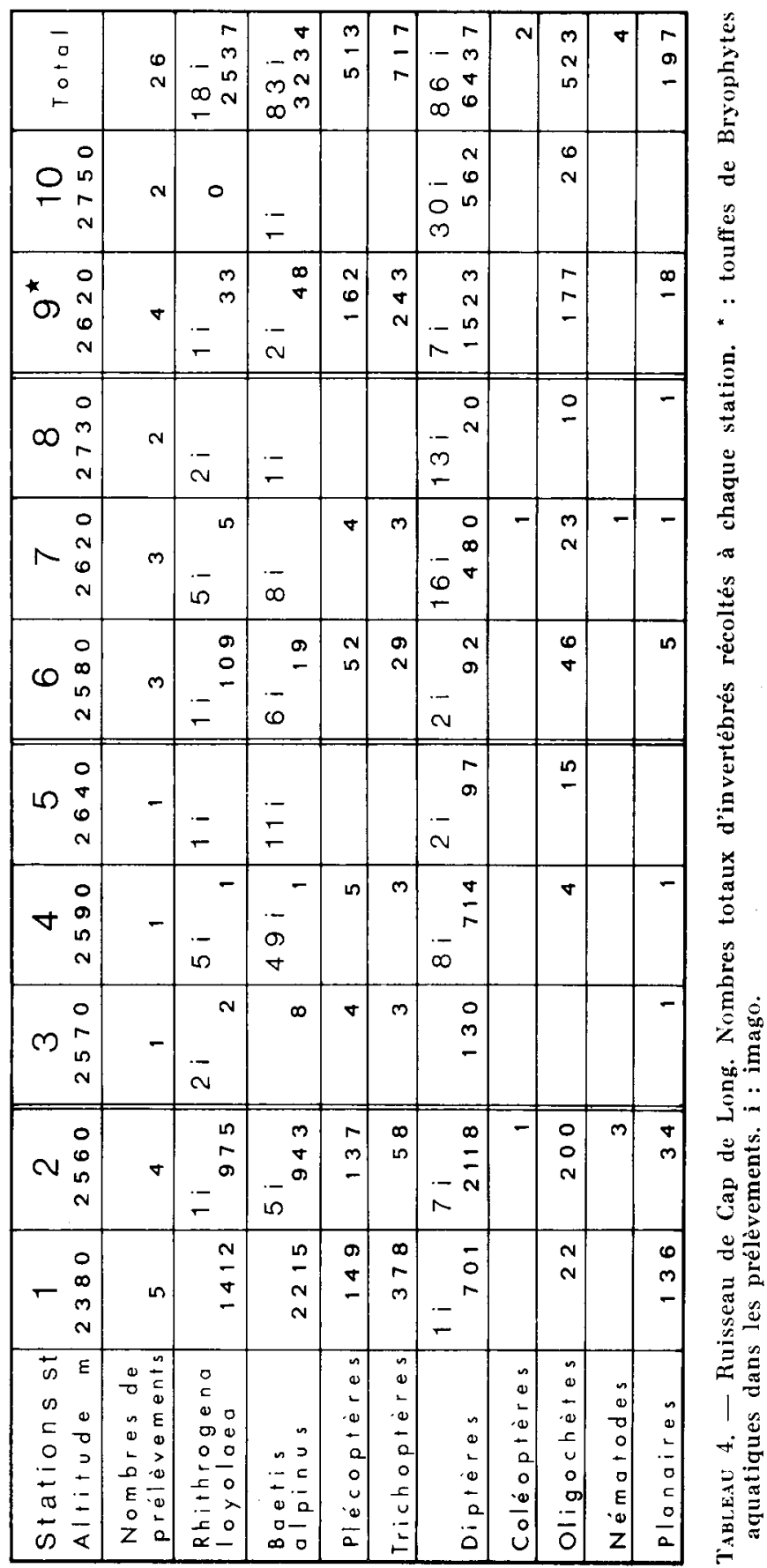




\begin{tabular}{|c|c|c|c|c|c|c|c|c|c|c|}
\hline Alt. $m$ & 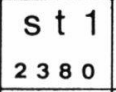 & \begin{tabular}{|l|}
$s t 2$ \\
2560 \\
\end{tabular} & $\begin{array}{l}s t 3 \\
2570\end{array}$ & $\begin{array}{r}s t 4 \\
2590\end{array}$ & $\begin{array}{l}s+5 \\
2640\end{array}$ & $\begin{array}{c}s+6 \\
2580\end{array}$ & $\begin{array}{lll}s & t & 7 \\
2 & 62 & 0\end{array}$ & 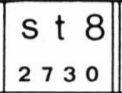 & 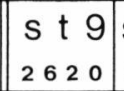 & $\begin{array}{l}s+10 \\
2750\end{array}$ \\
\hline \multicolumn{11}{|c|}{ Rhithrogena } \\
\hline $\begin{array}{c}\text { Eav }{ }^{\circ} \mathrm{C} \\
14.1 \times \\
1971\end{array}$ & 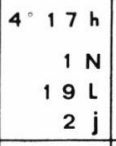 & $\begin{array}{r}4.16 \mathrm{~h} \\
15 \mathrm{~L}\end{array}$ & $\begin{array}{cccc}2 & 1 & 6 \mathrm{~h} \\
2 & \mathrm{i} & \\
& & \\
& 2 \mathrm{j}\end{array}$ & - & NEIGE & 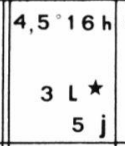 & $\begin{array}{r}3^{\circ} 15 \mathrm{~h} \\
2 \mathrm{j}\end{array}$ & $\begin{array}{l}1^{\circ} 15 \mathrm{~h} \\
1 \mathrm{i}\end{array}$ & $\begin{array}{rr}3{ }^{\circ} 16 \mathrm{~h} \\
& \\
4 & \mathrm{~L} \\
& 2 \mathrm{j}\end{array}$ & - \\
\hline $\begin{array}{l}14 . V I 1 \\
1972\end{array}$ & $\begin{array}{rrrr}1,5^{\circ} & 1 & 7 & h \\
5 & 4 & \mathrm{~L} \\
7 & 1 & \mathrm{j} \\
\end{array}$ & $N E I G E$ & $N E \perp G E$ & NEIGE & NEIGE & $N E \perp G E$ & $N E I G E$ & $N E \mid G E$ & $N E \backslash G E$ & $N E \perp G E$ \\
\hline $\begin{array}{l}9 . V 111 \\
1972 \\
\end{array}$ & - & $\begin{array}{rrrr}3,5^{\circ} & 1 & 5 & h \\
1 & 1 & 7 & L \\
& 4 & j\end{array}$ & $N E \mid G E$ & $N E \mid G E$ & $N E \mid G E$ & $\begin{array}{c}2,5^{\circ} 15 h \\
0\end{array}$ & NEIGE & $N E \mid G E$ & $\left|\begin{array}{r}2,516 h \\
1 L \star\end{array}\right|$ & NE I GE \\
\hline $\begin{array}{r}6.1 x \\
1972 \\
\end{array}$ & $\begin{array}{rrrr}3,5 & 1 & 7 & h \\
9 & 9 & \mathrm{~L} \\
3 & \mathrm{j}\end{array}$ & 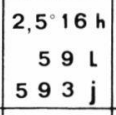 & $N E \mid G E$ & $N E \mid G E$ & $N E \perp G E$ & - & $\mid \begin{array}{c}2,5^{\circ} 15 \mathrm{~h} \\
1 \mathrm{i}\end{array}$ & $N E \mid G E$ & $\left|\begin{array}{ccc}2,5^{\circ} & 1 & 4 \\
3 & \mathrm{~h} \\
3 & \mathrm{j}\end{array}\right|$ & $\begin{array}{c}2,5^{\circ} 15 \mathrm{~h} \\
0\end{array}$ \\
\hline $\begin{array}{l}19 x \\
1972 \\
\end{array}$ & 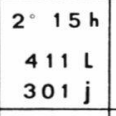 & - & - & - & - & - & - & & - & 一 \\
\hline $\begin{array}{c}7 a ̀ 14 \\
1 x \\
1973\end{array}$ & 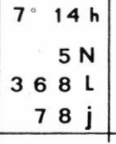 & $\begin{array}{llll}5 & 1 & 6 & h \\
1 & \mathrm{i} & & \\
& 6 & 4 & \mathrm{~L} \\
1 & 2 & 3 & \mathrm{j}\end{array}$ & $\begin{array}{c}3,5^{\circ} 18 \mathrm{~h} \\
-\end{array}$ & $\begin{array}{rr}2{ }^{2} & 14 \mathrm{~h} \\
5 & \\
& 1 \mathrm{j}\end{array}$ & $\begin{array}{l}1,517 h \\
1 i\end{array}$ & $\left|\begin{array}{llll}5,5 & 1 & 7 & h \\
1 & i & \\
3 & 0 & L & \star \\
7 & 1 & j\end{array}\right|$ & $\left|\begin{array}{ccc}4 & 17 \mathrm{~h} \\
4 & \mathrm{i} & \\
& 3 \mathrm{j}\end{array}\right|$ & $\mid \begin{array}{l}2,518 \mathrm{~h} \\
1 \mathrm{i}\end{array}$ & $\left|\begin{array}{cc}4 & 14 h \\
1 & i \\
17 & L \\
& \left(16^{\star}\right) \\
3 & j\end{array}\right|$ & $\begin{array}{c}0,5^{\circ} 17 \mathrm{~h} \\
0\end{array}$ \\
\hline \multicolumn{11}{|c|}{ Baetis } \\
\hline $\begin{array}{l}14-1 X \\
1971\end{array}$ & $\begin{array}{r}10 \mathrm{~L} \\
1 \mathrm{j} \\
\end{array}$ & $9 \mathrm{~L}$ & $8 \mathrm{j}$ & - & $N E \mid G E$ & $1 \mathrm{j}$ & 0 & 0 & $\begin{array}{l}1 \mathrm{~L} \star \star \\
2 \mathrm{j}\end{array}$ & - \\
\hline $\begin{array}{l}14-\mathrm{VII} \\
1972\end{array}$ & $\begin{array}{l}33 \mathrm{~L} \\
66 \mathrm{j}\end{array}$ & $N E \mid G E$ & $N E \mid G E$ & $N E \mid G E$ & $N E \mid G E$ & $N E \mid G E$ & $N E \mid G E$ & $N E \mid G E$ & $N E \mid G E$ & $N E \perp G E$ \\
\hline $\begin{array}{l}9-V 111 \\
1972 \\
\end{array}$ & - & $23 \mathrm{j}$ & $N E \mid G E$ & $N E \mid G E$ & NEIGE & $\mathbf{O}$ & NEIGE & $N E \mid G E$ & $\begin{array}{lll}1 & & \\
& 5 & j \\
\end{array}$ & NEIGE \\
\hline $\begin{array}{r}6-1 x \\
1972\end{array}$ & $\begin{array}{r}5 \mathrm{~N} \\
117 \mathrm{~L} \\
90 \mathrm{j} \\
\end{array}$ & $\begin{array}{rll}1 & \mathrm{i} & \\
4 & 0 \mathrm{l} \\
7 & 4 & 6 \mathrm{j}\end{array}$ & $N E I G E$ & $N E \mid G E$ & $N E \mid G E$ & - & $1 \mathrm{i}$ & $N E \mid G E$ & $\begin{array}{l}1 \mathrm{~L} \mathrm{~L}^{\star} \\
14 \mathrm{j}\end{array}$ & 0 \\
\hline $\begin{array}{l}19-X \\
1972 \\
\end{array}$ & 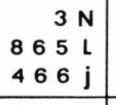 & - & - & 一 & - & - & - & - & - & - \\
\hline $\begin{array}{c}7 a 14 \\
1 x \\
1973\end{array}$ & $\begin{array}{rrrr}2 & 0 & 8 & N \\
3 & 3 & 5 & \mathrm{~L} \\
1 & 6 & \mathrm{j}\end{array}$ & 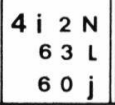 & - & $\begin{array}{r}49 \mathrm{i} \\
1 j\end{array}$ & $11 \mathrm{i}$ & 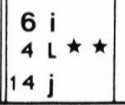 & $7 i$ & $1 \mathrm{i}$ & 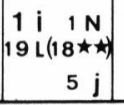 & $1 \mathrm{i}$ \\
\hline
\end{tabular}

Tableau 5. - Ruisseau de Cap de Long. Nombres d'imagos et larves d'Ephéméroptères récoltées dans chaque prélèvement. Longueurs (cerques non compris) : ${ }^{\star} \leqslant 8 \mathrm{~mm},{ }^{\star \star} \leqslant 5 \mathrm{~mm}$. Le trait vertical épaissi entre st 4 et st 5 (IX-1973) indique la présence d'une zone d'enneigement continue, d'environ $80 \mathrm{~m}$ de longueur sur le cours d'eau, immédiatement en amont de st 4 . Traits horizontaux : pas de prélèvements. 
Chez Baetis alpinus, le cycle de développement est complet à une altitude plus élevée (st. $2: 2560 \mathrm{~m}$ ). Les migrations minimales (observées sur 83 imagos) s'effectuent sur des distances atteignant $800 \mathrm{~m}$, avec un gain d'altitude de l'ordre de $170 \mathrm{~m}$. En réalité, les rares larves au dernier stade ( 2 seulement) récoltées à la station 2 ne peuvent expliquer le nombre d'imagos trouvées 'n amont, surtout si l'on tient comple de la grande fragilité des imagos d'Éphéméroptères : celles-ci ne peuvent séjourner longtemps dans une eau courante, en particulier sur un substrat aussi instable que les cailloutis de cirques glaciaires.

Aussi l'émergence de la plupart ou de la totalité des spécimens récoltés a-t-elle sans doute eu lieu en aval, c'est-à-dire au moins à la station 1, au bas de la cascade. Les distances parcourues pourraient alors dépasser $1 \mathrm{~km}$ et les gains d'altitude $350 \mathrm{~m}$.

En trois ans, une larve au dernier stade a été trouvée à la station 9, relativement peuplée (tableau 4) grâce à un substrat granitique stable, une exposition favorable et la présence de Bryophytes aquatiques.

On remarquera le très faible nombre de jeunes larves $\mathbf{j}$, dépourvues de fourreaux alaires, chez les deux espèces au-dessus de la station 2 au mois de septembre. Le temps d'incubation étant inconnu, l'éclosion peut ne pas avoir encore eu lieu. Mais il est également possible que, très près des névés, le mécanisme du cycle de colonisation ne serve pas à grand chose. Il apparaît surtout essentiel que la remontée des imagos et la ponte aient lieu à peu près à la limite de possibilité d'existence du cycle biologique de l'espèce, c'est-à-dire dans des zones régulièrement déneigées chaque année et assez tôt dans la saison (juin-juillet).

\section{V. - DISCUSSION}

1) Le nombre lotal d'imagos de Baetis récoltées sur les deux torrents étudiés est près de 10 fois supérieur (756) à celui des Rhithrogena (89) alors que les différences des densités de populations larvaires sont beaucoup plus faibles. Ceci peut s'expliquer par la pénétration active, connue depuis très longlemps (Eaton 1865), des q de Baetis dans l'eau lors de la ponte : elles ont ensuite beaucoup plus de chances d'être retenues, après leur mort, par les pierres du lit que les $q$ de Rhithrogena qui restent en surface.

2) Aucune imago ô de Baetis n'a été récoltée alors que les captures d’imagos of de Rhithrogena sur les pierres émergées humides de la source du Lavedan ne sont pas rares.

3 ) Une seule subimago migrante a été capturée (Baetis ô). Les déplacements des subimagos paraissent en général se limiter à 
quelques dizaines de mètres à la recherche d'un abri (arbustes, hautes herbes, etc.), en attendant la dernière mue. Les pièges de Roos (1957) et de Madsen et coll. (1973) ont également capturé peu de subimagos. Ces observations sur les Éphéméroptères rejoignent celles faites sur les Trichoptères par Roos (1957), Lehmann (1970) et Pearson et Kramer (1972) : les vols orientés vers l'amont intéresseraient surtout les individus matures. Ces résultals vont done netiement à l'encontre de l'une des conclusions de Dingle (1972) suivant laquelle les migrations auraient lieu avant l'ovogénèse.

4) Le mécanisme d'oviposition fonctionne même en eau très froide (moins de $3{ }^{\circ} \mathrm{C}$ ) puisque la quasi-totalité des $q$ étaient vides d'œufs. Les imagos de B. alpinus ne sont donc pas saisies par le froid en pénétrant dans l'eau.

5) L'éclosion dans une eau de température comprise toute l'année entre 0 et $3{ }^{\circ} \mathrm{C}$ n'a pu être prouvée chez $R$. loyolaea mais elle a lieu chez $B$. alpinus (tableau 1 ).

6) Les imagos de ces deux espèces paraissent attirées par le jaillissement de l'eau plutôt que par le flot lui-même : une partie des imagos au moins remonte ainsi le cours jusqu'à la source. En haute montagne, si la source est enneigée pendant la période de vol, l'oviposition a lieu immédiatement en aval de l'enneigement (Lavedan, le 6-VII-72, tableau 1). Une grande partie des imagos s'arrête ainsi en aval d'un obstacle assez important, même si le cours d'eau est dégagé en amont (Cap de Long, enneigement sur $80 \mathrm{~m}$ entre les st. 4 et 5 en IX-1973, tableau 5). De la même façon, la naissance d'un déversoir de lac de montagne constitue un lieu de ponte recherché par les Ephéméroptères : j'ai récolté des imagos $q$ dans les premiers mètres du lit des r. de Lassas vers $2500 \mathrm{~m}$ et de Sarrouyès à $2170 \mathrm{~m}$, tous deux affluents de la Neste d'Aure. Ce dernier point est en accord avec les observations de Roos (1957) et d'Ulfstrand (1970) sur les vols de Trichoptères ainsi qu'avec celles de Müller (1954) et celles de Wood rapportées par Mackay (1969) sur les Simulies. Les Ephéméroptères (Harris 1952) et les Trichoptères (Roos 1957) pondent volontiers dans les rapides situés entre des zones d'eau calme.

Les stimuli déterminant l'arrêt de la progression des imagos et en particulier des Ephéméroptères seraient ainsi, au moins en partie, de nature visuelle (reflets lumineux ?).

Verrier $(1954,1956)$ a signalé des migrations de l'Ephéméroptère Polymitarcis virgo en direction du soleil couchant. Dans les deux cas étudiés ici, la remontéc a lieu aussi - très approximativement toutefois - en direction du soleil couchant.

Le thermotropisme éventuel invoqué par Verrier $(1954,1956)$ n'est pas exclu dans certains cas. Il est possible qu'il intervienne 
chez les insectes sténothermes lors de brusques changements de température, à l'arrivée d'un affluent par exemple (Roos 1957). Selon Bogoescu (1970), les contacts répétés de la partie postérieure de l'abdomen de l'Éphéméroptère avec la surface de l'eau permettraient à l'insecte d'évaluer la température de celle-ci et d'orienter ses déplacements.

7) L'action du vent mériterait une étude particulière : elle paraît très variable selon la force de celui-ci.

Un vent assez fort entraîne la majorité des imagos (Brindle 1357, Elliott 1967). Cependant, cette action est certainement assez limitée car beaucoup d'insectes volent surtout par temps calme et se mettent le plus possible à l'abri au-delà d'une certaine vitesse du vent. Tout vol cesse pour une vitesse du vent de l'ordre d'un $\mathrm{m} / \mathrm{s}$, soit seulement $3,6 \mathrm{~km} / \mathrm{h}$, chez deux espèces de Diptères Chloropidae (Dorner et Mulla 1962, Gerhardt et Axtell 1972) et pour une vitesse voisine de $5 \mathrm{~m} / \mathrm{s}$ chez l'Ephéméroptère Stenonema canadense (Thew 1958).

Par contre, les imagos peuvent lutter contre un vent faible et se déplacent fréquemment face à lui (Spieth 1940, Harris 1952, Verrier 1954 et 1956, Roos 1957, Bishop 1973, etc.).

D'autres auteurs enfin, ont observé des déplacements d'imagos, d'Ephéméroptères notamment, vers l'amont quelle que soit la direction du vent (Madsen et coll. 1973, et en particulier Russev 1970). Dans ce cas, les vitesses du vent, non mesurées, sont vraisemblablement faibles.

En haute montagne, par beau temps l'été, l'insolation provoque un réchauffement des masses d'air, plus denses dans les vallées, qui se traduit, surtout l'après-midi, par un courant ascendant. Au niveau du sol, ce déplacement d'air est le plus souvent imperceptible ou faible; il intervient vraisemblablement mais il est douteux qu'il constitue la cause essentielle du type de migration observé ici. Par temps agité, les rafales de vent, sont, à cause du relief tourmenté, le plus souvent soudaines et brutales, incompalibles avec le vol des insectes. Quant au très faible déplacement d'air, dirigé vers l'aval et provoqué par le frottement de l'eau sur les couches d'air, il n'a certainement aucune influence, à cause de l'altitude de vol des Ephéméroptères.

8) Cette étude a été limitée aux Ephéméroptères mais à haute altilude, certains Plécoptères migrent également vers l'amont. Ainsi, les femelles d'Isoperla viridinervis Pictet (déterminations C. Berthélemy) sont nombreuses en septembre à la source du Lavedan, alors qu’à la même époque les larves n’apparaissent qu'à 100$150 \mathrm{~m}$ plus bas et ne sont réellement abondantes qu'à $300 \mathrm{~m}$ en aval. Ceci va dans le sens des observations de Schwarz (1970) sur Isoperla goertzi. 


\section{Remarque.}

32 Planaires ont été dénombrées au contact des imagos d'Éphéméroptères, la plupart à la source du Lavedan. Elles appartiennent loutes à l'espèce Crenobia alpina (Dana) quoique Polycoelis felina (Dalyell) soit également présente, en nombre moindre, dans les prélèvements (déterminations M. Lafont). Les Ephémères adultes étaient la plupart du temps déjà vidés de leurs œufs, mais il demeure une possibilité de prédation à leurs dépens au moment de la ponte dans ces eaux très froides faiblement peuplées. Crenobia alpina s'attaque aux membranes articulaires de l'insecte et fait pénétrer son pharynx par des déchirures de celles-ci. L’introduction du pharynx des gros specimens a lieu plus particulièrement entre les sternites abdominaux, ou entre le premier tergite abdominal et le métathorax ou encore entre les pièces latérales du thorax. Les petits spécimens pénètrent souvent tout entiers à l'intérieur du corps de l'Ephémère mais peuvent aussi introduire leur pharynx dans des éléments de diamètre réduit (pattes, en général par l'articulation fémur-tibia).

\section{VI. - CONCLUSION}

L'hypothèse du «Cycle de colonisation» (Müller 1954) est sans aucun doute valable, au moins dans certaines conditions. Néanmoins, l'existence et l'étendue éventuelle de ce processus varient selon les groupes taxonomiques. Son efficacité dépend des caraclères de chaque cours d'eau (en parliculier altitude, pente, longueur, largeur, profondeur moyenne, nature du fond et des rives, existence ou non de zones d'eau stagnante, végétation sur les bords el au-dessus du cours d'eau, régime des vents). Ses rapports avec la dérive et avec les remontées de larves vers l'amont dépendent probablement aussi des mêmes facteurs (Waters 1972).

Ce mécanisme est sans doute très important dans les pelits ruisseaux et torrents froids des régions montagneuses (Roos 1957, Schwarz 1970, Madsen et coll. 1973).

Les remontées de larves d'Éphéméroptères sont lentes en effet. Sur le substrat instable de certains cirques glaciaires, elles sont susceptibles d'être compromises ou anihilées en quelques minules par de violentes crues, alors que le cycle de colonisation peut s'opposer efficacement à la dérive. Dans ces conditions extrêmes, on peut parler, au sujet du peuplement, d'un « front de colonisation fluctuant». Selon Pearson et Kramer (1972), qui ont étudié un torrent moins froid, la migration des adultes vers l'amont pour la ponte correspondrait à la recherche de conditions privilégiées 
(sources). Cette interprétation ne peut être retenue ici, en particulier pour le second cours d'eau.

En définitive, bien qu'il présente encore beaucoup d'inconnues, notamment en ce qui concerne ses limites, le concept du cycle de colonisation de Müller (1954) demeure donc séduisant. Il est cité dans les manuels récents d'écologie des eaux courantes (Hynes 1970 a, Décamps 1971) et Hynes lui attribuc même une grande importance dans certains cas : "Insects which have actively flying adults have a further method of regaining ground lost by drifting, and this may be one of the reasons why they so often dominate the fauna of small streams ... ». Cette idée s'applique particulièrement bien aux ruisseaux de haute montagne, dans les Pyrénées.

\section{TRAVAUX GITÉS}

Aubert (J.), Goeidi.in (P.) et Lyon (J.-P.). 1969. - Essais de marquage et de reprise d'insectes migrateurs en automne 1968. Mitt. schweiz. ent. Ges., $42(1 / 2): 140-166$.

Bengtsson (J.), Butz (I.) et Madsen (B. L.). 1972. - Opstrømsflugt hos vandløbsinsekter. Flora Fauna, 78 (4) : 102-104.

Bishop (J. E.). 1973. - Limnology of a small Malayan River Sungai Gombak. Junk Publ., 485 p., The Hague.

Bogoescu (C.). 1970 (1973). - Recherches concernant l'éthologie et l'écologie des Éphéméroptères. Considérations concernant le vol pour la ponte et le déterminisme de l'aire de répartition des Ephéméroptères. Proc. First Int. Conf. Ephemeroptera. Brill, 199-204, Leiden.

Bogoescu (C.) et Tabacaru (I.). 1969. - Observațiuni asupra zborului nuptial şi acuplării la Ephemeroptere. Communicari Zoologie Bucarest, $1: 78-84$.

Brindee (A.). 1957. - Effect of temperature and humidity on the flight of Trichoptera. Entomologist's mon. Mag., $93: 63-66$.

Chipman (J. A.) et KinghorN (J. M.). 1955. - Window-trap for flying insects. Can. Ent., $82: 46-47$.

Davies (D. M.), Peterson (B. V.) et Wood (D. M.). 1961 (1962). - The Black Flies (Diptera : Simuliidae) of Ontario. Part. I. Adult identification and distribution with descriptions of six new species. Proc. ent. Soc. Ont., 92 : 70-154.

Decamps (H.). 1971. - La vie dans les cours d'eau. Presses Universitaires de France, 128 p., Paris.

Dingle (H.). 1972. - Migration Strategies of Insects. Science, N.Y., $175: 1327-1335$.

Dorner (R. W.) et Mulla (M. S.). 1962. - Laboratory study of wind velocity and temperature preference of Hippelates eye gnat. Ann. ent. Soc. Amer., $55: 36-39$.

Dorris (T. C.) et Copeland (B. J.). 1962. - Limnology of the middle Mississipi river. III. Mayfly populations in relation to navigation water level control. Limnol. Oceanogr., $7: 240-247$.

Dumont (H. J.) et Hinnekint (B. O. N.). 1973. - Mass migration in dragonflies, especially in Libellula quadrimaculata L. : a review, a new ecological approach and a new hypothesis. Odonatologica, (2) $1: 1-20$. 
Eaton (A. E.). 1865. - Occurence of the female imatgo of Clöeon under submerged stones. Entomologist's mon. Mag., 2 : 14-15.

EINSELE (W.). 1960. — Die Strömungsgeschwindigkeit als beherrschender Faktor bei der limnologischen Gestaltung der Gewässer. Oesterr. Fish. Suppl. 1, $2: 40$ p. (non vu).

El.liotT (J. M.). 1967. - Invertebrate drift in a Dartmoor stream. Arch. Hydrobiol., 63 (2) : 202-237.

EiliotT (J. M.). 1969. - Life history and biology of Sericostona personatum Spence (Trichoptera). Oikos, $20: 110-118$.

Elaiott (J. M.). 1971. -- Life histories and drifting of three species of Limnephilidae (Trichoptera). Oikos, $22: 56-61$.

Ferago. 1937. - La pêche à la truite à la mouche de mai. Pêche illustrée, $193: 122$ (non vu).

Gerhardt (R. R.) et Axtell (R. C.). 1972. - Flight of the eye gnat, Hippelates pusio (Diptera : Chloropidae) : effect of temperature, light, moisture and wind velocity. J. med. Ent., 9 (5) : 425-428.

Harris (J. R.). 1952. - An Angler's Entomology. Collins, 268 p., London.

HyNes (H. B. N.). 1970 a. - The Ecology of Running Waters. Liverpool University Press, 555 p.

Hynes (H. B. N.). 1970 b. - The ecology of stream insects. A. Rev. Ent, $15: 25-42$.

Lehmans (U.). 1970. - Stromaufwärts gerichteter Flug von Philopotamus montanus (Trichoptera). Oecologia, 4 (2) : 163-175.

Lestage (J. A.). 1937. - Contribution à l'étude des 'Éphéméroptères. XIV. Quelques cas de pontes aberrantes. Bull. Annls Soc. r. ent. Belg., $77: 332-336$.

Macan (T. T.). 1957. - The Life Histories and Vigrations of the Ephemeroptera in a Stony Stream. Trans. Soc. Br. Ent., 12 (5) : 129-156.

Mackay (R. J.). 1969. - Aquatic Insect Communities of a Small Stream on Mont St. Hilaire, Quebec. J. Fish. Res. Bd Can., 26 (5) : 1157 1183.

Madsen (B. L.), Bengtson (J.) et Butz (I.). 1973. - Observations on upstream migration by imagines of some Plecoptera and Ephemeroptera. Limnol. Oceanogr., 18 (4) : 678-681.

Malaise (R.). 1937. - A new insect-trap. Ent. Tidskr. $58: 148-160$.

Mulder (K.). 1954. - Investigations on the Organic Drift in North Swedish Streams. Rep. Inst. Freshwat. Res. Drottningholm, 35 : 133-148.

Mulder (K.). 1973. - Life cycles of stream insects. Aquilo, Ser. Zool., $14: 105-112$.

Muller-Limbeniu (I.). 1969. - Revision der europäischen Arten der Gattung Baetis Leach, 1815 (Insecta, Ephemeroptera). Gewäss. Abwäss., 48/49:1-214.

Muttkowski (R. A.). 1929. - The ecology of trout streams in Yellowstone National Park. Roosevelt wild Life Ann., 2 : 155-240 (non vu).

Nishimura (N.). 1967. - Ecological study on net spinning caddisfly, Stenopsyche griseipennis Mc Lachlan. II. Upstream-migration and determination of flight distance. Mushi, 40 (4) : 39-46.

Pearson (W. D.) et Kramer (R. H.). 1972. - Drift and Production of Two Aquatic Insects in a Mountain Stream. Ecol. Monogr., 42 (3) : $365-385$.

Roos (T.). 1957. - Studies on Upstream Migrations in Adult StreamDwelling Insects. I. Rep. Inst. Freshwat. Res. Drotlningholm, $38: 167-193$. 
Russev (B.). 1959. - « Vol de compensation pour la ponte» de Palingenia longicauda Oliv. (Ephem.) contre le courant du Danube. C.r. Acad. bulg. Sci., 12 (2) : 165-168.

Russev (B. K.). 1970 (1973). - Kompensationsflug bei der Orinung Ephemeroptera. Proc. First Int. Conf. Ephemeroptera. Brill, 132142. Leiden.

Sawyer (F. E.). 1950. - Studies of the sherry spinner. Salm. Trout Mag., $129: 110-114$.

Schoexemuid (E.). 1930. - Eintagsfliegen oder Ephemeroptera. In Dahls «Die Tierwelt Deutschlands», $19: 106$ p. Jena.

Schumacher (H.). 1970. - Untersuchungen zur Taxonomie, Biologie und Oekologie einiger Köcherfliegenarten der Gattung Hydropsyche Pict. (Insecta, Trichoptera). Int. Revue ges. Hydrobiol., 55 (4) : 511-557.

SchWarz (P.). 1970. - Autökologische Untersuchungen zum Lebenszyklus von Setipalpia-Arten (Plecoptera). Arch. Hydrobiol., 67 (1) : 103140, 67 (2) : 141-172.

Schwoenber, (J.). 1969. - Oekologie der Süsswassertiere Fliessgewässer. Fortschr, Zool., 20 (2) : 173-206.

SpIEtr (H. T.). 1940. - Studies on the biology of Ephemeroptera. II. The nuptial flight. $J l$ N.Y. ent. Soc., $48: 379-390$.

Svensson (B. W.). 1974. - Population movements of arlult Trichoptera at a South Swedish stream. Oikos, $25: 157-175$.

THEw (T. B.). 1958. - - Studies on the mating flights of the Ephemeroptera I. The mating flights of Ephoron album (Say) and Stenonema canadense (Walker). Fla Enl., 41 : 9-12.

Thomas (A.). 1970. - Sur la taxonomie de deux Rhithrogena des Pyrénées (Ephemeroptera, Heptageniidae). Annls Limnol., (6 (3) : 305-315.

Thomas (E.). 1966. - Orienticrung der Imagines von Capnia atra Morton (Plecoptera). Oikos, 17 : 278-280.

Ulfstrand (S.). 1970. - Trichoptera from River Vindelälven in Swedish Lapland. Ent. Tidsk., $91: 46-63$.

Ulmer (G.). 1927. - Verzeichnis der deutschen Ephemeropteren und ihrer Fundorte. Konowia, 6 : 234-262.

Verrier (M. L.). 1941. - Pontes aberrantes chez les Ephémères et conséquences biologiques. C. r. Séanc. Acad. Sci., 3-XI-1941, 213 : 603-632.

Verrier (M. L.). 1954. - Rassemblements et migrations chez les Ephémères. Bull. biol. Fr. Belg., 88 : 68-89.

Verrier (M. L.). 1956. - Biologie des Ephémères. Armand Colin, 216 p., Paris.

Waters (T. F.). 1965. - Interpretation of invertebrate drift in streams. Ecology, $46: 327-334$.

Waters (T. F.). 1968. - Diurnal periodicity in the drift of a day active stream invertebrate. Ecology, 49 (1) : 152-153.

Waters (T. F.). 1972. - The drift of stream insects. A. Rev. Ent., 17 : 253-272. 